\title{
Phenotypic Detection of Carbapenemase Production in Carbapenem-Resistant Enterobacteriaceae by Modified Hodge Test and Modified Strip Carba NP Test
}

\author{
Nisha Patidar ${ }^{1}$ Nitya Vyas ${ }^{1}$ Shanoo Sharma ${ }^{1}$ Babita Sharma ${ }^{1}$ \\ ${ }^{1}$ Department of Microbiology, SMS Medical College, Jaipur, \\ Rajasthan, India \\ Address for correspondence Babita Sharma, MD, 31 Shree Ganesh \\ Colony Mahesh Nagar, 80 Feet Road, Jaipur 302019, Rajasthan, India \\ (e-mail: babitasharma13@yahoo.com). \\ Nisha Patidar, MD, B-7; L-1126, Vrinda Gardens, Jagatpura, Jaipur \\ 302017, Rajasthan, India (e-mail: sweetdr.nisha@gmail.com).
}

J Lab Physicians 2021;13:14-21.

\author{
Abstract \\ Keywords \\ - carbapenem-resistant \\ Enterobacteriaceae \\ - carbapenemase \\ producing \\ Enterobacteriaceae \\ - modified Hodge test \\ - modified strip Carba \\ NP test
}

Objective Carbapenems are last resort antibiotics for multidrug-resistant Enterobacteriaceae. However, resistance to carbapenem is increasing at an alarming rate worldwide leading to major therapeutic failures and increased mortality rate. Early and effective detection of carbapenemase producing carbapenem-resistant Enterobacteriaceae (CRE) is therefore key to control dissemination of carbapenem resistance in nosocomial as well as community-acquired infection. The aim of present study was to evaluate efficacy of Modified strip Carba NP (CNP) test against Modified Hodge test (MHT) for early detection of carbapenemase producing Enterobacteriaceae (CPE).

Material and Methods Enterobacteriaceae isolated from various clinical samples were screened for carbapenem resistance. A total of 107 CRE were subjected to MHT and Modified strip CNP test for the detection of CPE.

Statistical Analysis It was done on Statistical Package for the Social Sciences (SPSS) software, IBM India; version V26. Nonparametric test chi-square and Z-test were used to analyze the results within a $95 \%$ level of confidence.

Results Out of 107 CRE, 94 (88\%) were phenotypically confirmed as carbapenemase producer by Modified strip CNP test and 46 (43\%) were confirmed by Modified Hodge Test (MHT). Thirty-eight (36\%) isolates showed carbapenemase production by both MHT and CNP test, 56 isolates (52\%) were CNP test positive but MHT negative, eight (7\%) isolates were MHT positive but CNP test negative and five (5\%) isolates were both MHT and CNP test negative. There is statistically significant difference in efficiency of Modified CNP test and MHT $(p<0.05)$.

Conclusion Modified strip CNP test is simple and inexpensive test which is easy to perform and interpret and gives rapid results in less than 5 minutes. It has high degree of sensitivity and specificity. Modified strip CNP test shows significantly higher detection capacity for carbapenemase producers as compared with MHT. published online May 19, 2021
DOI https://doi.org/

10.1055/s-0041-1723859 ISSN 0974-2727. (c) 2021. The Indian Association of Laboratory Physicians.

This is an open access article published by Thieme under the terms of the Creative Commons Attribution-NonDerivative-NonCommercial-License, permitting copying and reproduction so long as the original work is given appropriate credit. Contents may not be used for commercial purposes, or adapted, remixed, transformed or built upon. (https://creativecommons.org/licenses/by-nc-nd/4.0/).

Thieme Medical and Scientific Publishers Pvt. Ltd. A-12, 2nd Floor, Sector 2, Noida-201301 UP, India 


\section{Introduction}

Members of Enterobacteriaceae family are gram negative, rodshaped facultative anaerobes mainly colonizing the intestinal tracts of humans and animals and are a common cause of community-associated as well as health care-associated infections. ${ }^{1}$

In the past, most of the first line and low-cost antimicrobial drugs such as penicillin and first and second generation cephalosporins have been used effectively for the management of gram-negative bacterial infections. However, drug-resistant organisms acquire resistance to these first-line antibiotics, thereby necessitating the need of second-line drugs like the third and fourth generation cephalosporins. Further emergence of $\beta$ lactamases, extended spectrum $\beta$ lactamases (ESBL), ${ }^{2}$ and AmpC $\beta$ lactamases ${ }^{3}$ producing bacterial strains lead to the development of multidrug-resistant (MDR) Enterobacteriaceae.

Carbapenems such as ertapenem, imipenem, meropenem, and doripenem have proven efficacy in severe infections caused by these MDR Enterobacteriaceae; therefore, they are frequently used as last resort therapeutic options. Carbapenems have the widest spectrum of antibacterial activities ${ }^{4}$ and are also active against the chromosomal cephalosporinases and ESBL, therefore are preferred antibiotics in case of invasive or life-threatening infections. However, in recent years, carbapenem-resistant Enterobacteriaceae (CRE) has emerged in the community as a major health threat.

Carbapenem resistance was first reported sporadically in the mid-1990s from the United States and since then reports of carbapenem resistance outbreaks are increasing worldwide at an alarming rate. ${ }^{5}$ In addition to carbapenem resistance, CRE often carry genes that confer high levels of resistance to many other antimicrobials, therefore these bacteria are difficult to treat and are associated with high mortality. ${ }^{6}$. The mechanisms of development of carbapenem resistance in Enterobacteriaceae are complex because they involve a broad range of organisms and are mediated by different mechanisms. Broadly, CRE can be carbapenemase producing CRE (CP-CRE) or noncarbapenemase producing CRE (non-CP-CRE). ${ }^{7}$

CP-CRE also known as carbapenemase producing Enterobacteriaceae (CPE) encompasses organisms that are resistant to carbapenems due to the production of a carbapenemase enzyme. Carbapenemases are specific $\beta$-lactamases enzymes produced by gram-negative bacteria having ability to hydrolyze carbapenems and other $\beta$-lactam antibiotics like penicillin and cephalosporins. Various carbapenemases have been reported in Enterobacteriaceae which can be classified as Class A: plasmid encoded Klebsiella pneumoniae carbapenemase (KPC) and Guiana extended spectrum variant, ${ }^{8}$ Class B metallo- $\beta$-lactamase (MBL) including imipenemases, Verona integron-encoded metallo- $\beta$-lactamase (VIM), New Delhi metallo- $\beta$-lactamases (NDM), ${ }^{8-10}$ Class D Oxacillinase (OXA) comprises OXA-48 derivatives. ${ }^{11}$ India is endemic for NDM type MBL while KPC producing isolates are sporadic. ${ }^{12-14}$

Noncarbapenemase producing carbapenem-resistant Enterobacteriaceae (non CP-CRE) encompasses organisms that are resistant to carbapenems due to mechanisms other than carbapenemase production like increase in bacterial outer membrane permeability, augmenting drug efflux, mutations or loss of outer membrane porins preventing entry of antibiotics, or due to overexpression of $\beta$-lactamases like ESBL and AmpC $\beta$-lactamases. ${ }^{7,15}$

Noncarbapenemase-related mechanism of carbapenem resistance are nontransferable while carbapenemase production is plasmid encoded. Thus, CPE have potential for widespread transmission, resulting in increased frequency of carbapenem resistance on a worldwide basis. ${ }^{8}$

Currently, CRE are a problem not confined to individual facilities but are affecting entire communities and nations. High incidence of treatment failures, morbidity, and mortality in many infectious diseases are strongly correlated with carbapenem resistance. There are limited antimicrobials effective against carbapenemase-producing bacteria. Most hospitals have limited facilities and resources to properly confirm the presence of carbapenemase production in clinical isolates. This is the need of hour to have a laboratory test which is easy to perform, cost effective, and at the same time is highly sensitive and specific. Genotypic methods like real time polymerase chain reaction-restricted fragment length polymorphism (PCR-RFLP) $)^{16,17}$ and whole genome sequencing, and other techniques like matrix assisted laser desorption ionization-time of flight-mass spectrometry $\left(\right.$ MALDI-TOF-MS) ${ }^{18,19}$ have overall good sensitivities and specificities but require trained microbiologists, expensive equipment, and are time consuming. In addition, it may fail to detect unknown novel carbapenemase genes. ${ }^{20}$ On the other hand, phenotypic tests like Modified Hodge test (MHT) and Carba NP (CNP) test are inexpensive, easier to perform, and require minimal training. MHT is highly sensitive test for detecting KPC and OXA-48 producers but has low sensitivity for MBL producers ${ }^{21,22}$ and low specificity due to false positives from ESBL or AmpC $\beta$-lactamase producers with porin loss. ${ }^{22-24}$ Also, it requires long incubation period of 24 hours. CNP test is a rapid chromogenic test which gives results in 2 hours with approximately $100 \%$ sensitivity and specificity for detecting carbapenemases in Enterobacteriaceae. But its cumbersome procedure requires bacterial extract. Modified strip CNP test is a modification of the CNP test and can be performed directly on bacterial culture instead of bacterial extract. The sensitivity and specificity of this test is reported to be equivalent to the original CNP test..$^{25,26}$

The objective of present study was to evaluate the modified strip CNP test against the MHT for detection of carbapenemase production on CRE isolates.

\section{Materials and Methods}

Present study was performed in the Microbiology department of tertiary care teaching hospital of North India from September 2018 to August 2019 with due permission from Institutional Ethical Committee.

Various clinical specimens like pus, blood, sputum, throat swab, endotracheal tube aspirate, CSF, urine, body fluids, etc. obtained from various medical and surgical wards, ICU 
and OPD were included in the study. These samples were further processed for identification of organism as per routine laboratory protocols. Organisms identified as members of Enterobacteriaceae were screened for carbapenem resistance by Kirby Bauer disk diffusion method following CLSI 2018 guidelines using Meropenem $10 \mu \mathrm{g}$ disk. Isolates which show inhibition zone diameter of $\leq 19 \mathrm{~mm}$ were considered as screening test positive and named as carbapenem-resistant Enterobacteriaceae (CRE).

One hundred seven CRE isolates were further evaluated for carbapenemase production by MHT and Modified strip CNP test.

$\mathrm{MHT}^{27}$ is performed as per recommendations of CDC. MHT Positive test has a clover leaf-like indentation of the Escherichia coli 25922 growing along the test organism growth streak within the disk diffusion zone.

Modified strip CNP test ${ }^{25}$ is a colorimetric test based on detection of hydrolysis of the $\beta$-lactam ring of a carbapenem (imipenem) by carbapenemase enzyme produce by bacteria. Hydrolysis of imipenem acidifies the medium, changing the color of the $\mathrm{pH}$ indicator (phenol red solution) from red to yellow. In present study, CNP test was modified by using paper strip as medium. In addition, pharmaceutical imipenem + cilastatin powder was used as a substrate in place of standard imipenem powder further reducing the cost. This test was performed directly on bacterial culture instead of bacterial extract making it easy as compared with original CNP test. Reagents used were same as original CNP test; Solution A (phenol red, $\mathrm{ZnSO}_{4} .7 \mathrm{H}_{2} \mathrm{O}, \mathrm{pH} 7.8$ ) and Solution B (Solution A $+12 \mathrm{mg} / \mathrm{mL}$ of imipenem). A Whatman filter paper is cut into square strips of size $15 \times 15 \mathrm{~mm}$. One strip was labeled as control strip and other as test strip. Control strip was moistened with $50 \mu \mathrm{L}$ of solution A while Test strip was moistened with $50 \mu \mathrm{L}$ of solution B. Subsequently one calibrated loop $(1 \mu \mathrm{L})$ of test organism grown on Mueller Hinton agar at $37^{\circ} \mathrm{C}$ for 18 to 24 hours was directly applied on the control and test strip by rubbing in circular fashion of approximately 5 to $7 \mathrm{~mm}$ in diameter. Results were read in 1 to 5 minutes. Isolates giving yellow color on the test strip with more intensity than on the control strip were considered as carbapenemase producers ( - Fig. 1).

K. pneumoniaeATCC BAA1705 strain was used as positive control and $K$. pneumoniae ATCC BAA1706 as negative control.

\section{Statistical Analysis}

Statistical Package for the Social Sciences (SPSS) software, IBM India; version V26 was used for statistical analysis. The data collected was qualitative and expressed as proportions or percentage. To ensure the results obtained are statistically significant within a 95\% level of confidence (with $p$-value 0.05), systematic hypothesis tests like $Z$-test and Chi-square test have been performed. Results were analyzed to determine if there was any significant difference in efficiency of MHT and Modified strip CNP test for early detection of CPE. Crosstabs were used to demonstrate the relationship

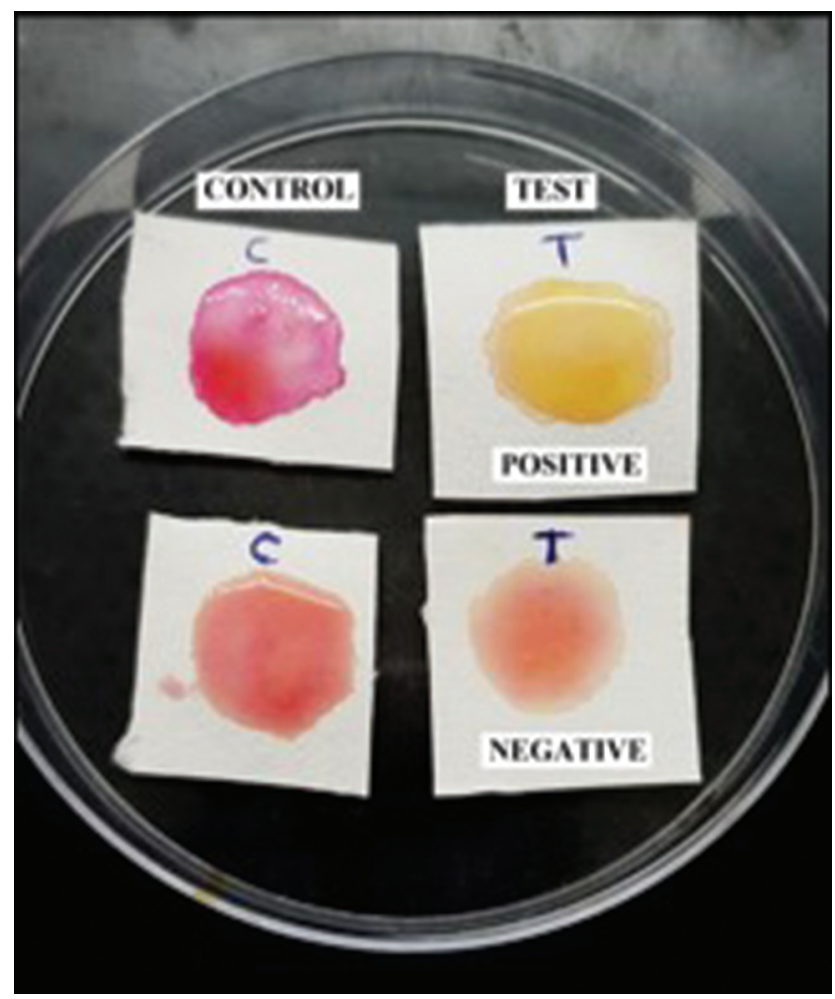

Fig. 1 Modified strip Carba NP test.

between the two test types MHT and Modified strip CNP test. Cohen's kappa coefficient $(\boldsymbol{k})$ was used to measure inter-rater reliability (measure of agreement between two test types).

\section{Results}

A total of 107 CRE isolates were included in this study. Majority of CRE was isolated from age group 21 to 40 years (36\%) followed by 41 to 60 years (23\%) with male preponderance over female patients ( - Fig. 2). Maximum number of CRE were isolated from samples received from wards 55 (52\%) followed by intensive care unit (ICU) 27 (25\%) and outdoor units 25 (23\%). CRE was isolated in maximum number from urine samples 44 (41\%) followed by ET secretion $27(25 \%)$ (-Fig. 3).

Out of 107 CRE isolated, the most common species isolated were Enterobacter species 50 (47\%) followed by E. coli 32 (30\%), Klebsiella species 18 (17\%), Citrobacter species 4 (4\%), and Proteus species 3 (3\%) (-Table 1).

Out of 107 CRE isolates, 46 (43\%) isolates were phenotypically confirmed as carbapenemase producer by MHT and 94 (88\%) were confirmed by Modified strip CNP test (at significance level of $\alpha=0.5$, Chi-square value with 1 degree of freedom is $47.592, Z$-value is $6.89, p$-value $<0.05$ for both Chi-square test and Z-test, significantly) (- Table 1; - Fig. 4), 38 (36\%) isolates showed carbapenemase production by both MHT and CNP test, five (5\%) isolates were both MHT and CNP test negative, eight (7\%) were MHT positive but CNP test negative and 56 (52\%) were MHT negative but CNP test positive (-Table 2). Measure of agreement (kappa value) 


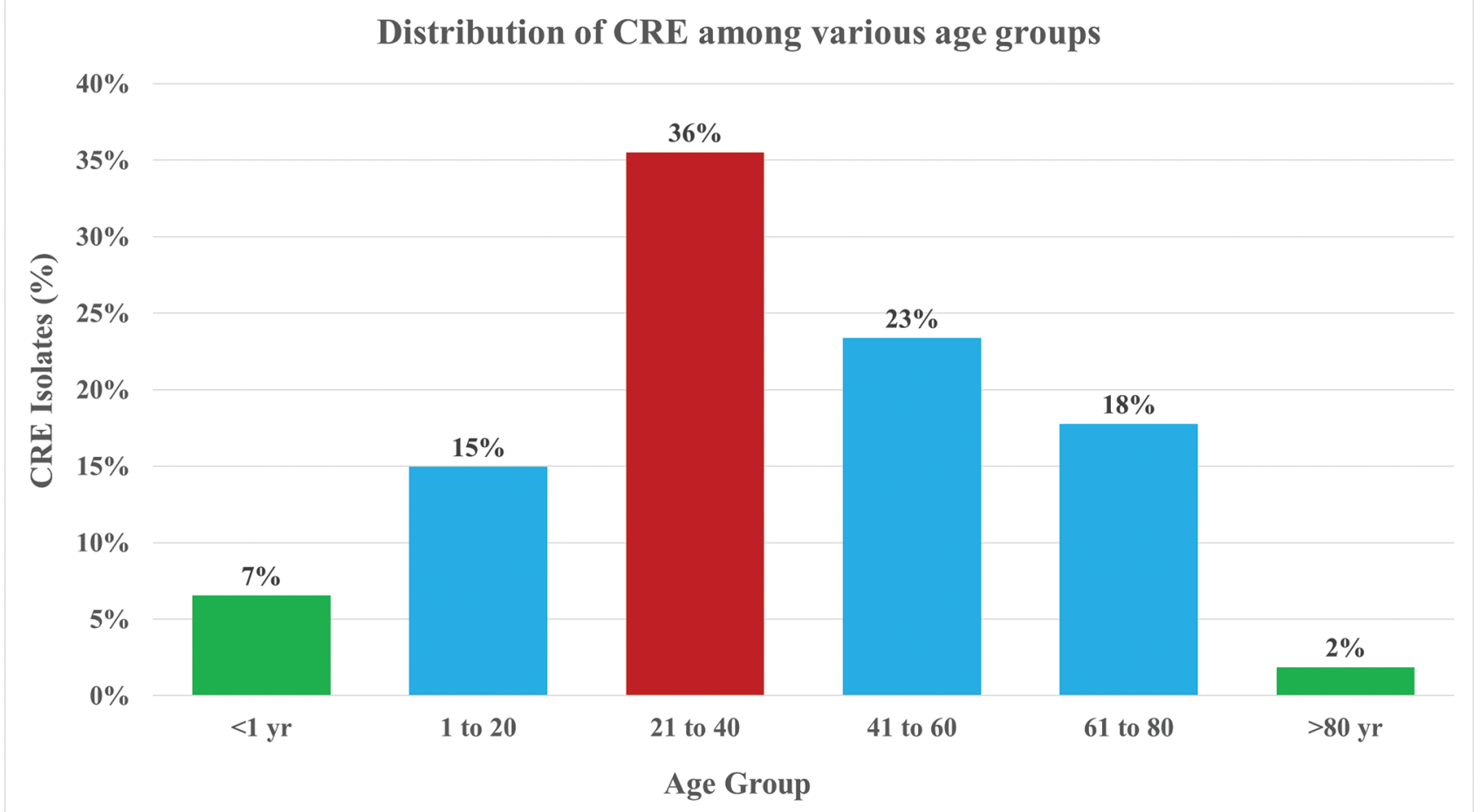

Fig. 2 Distribution of CRE among various age groups. CRE, carbapenem-resistant Enterobacteriaceae.

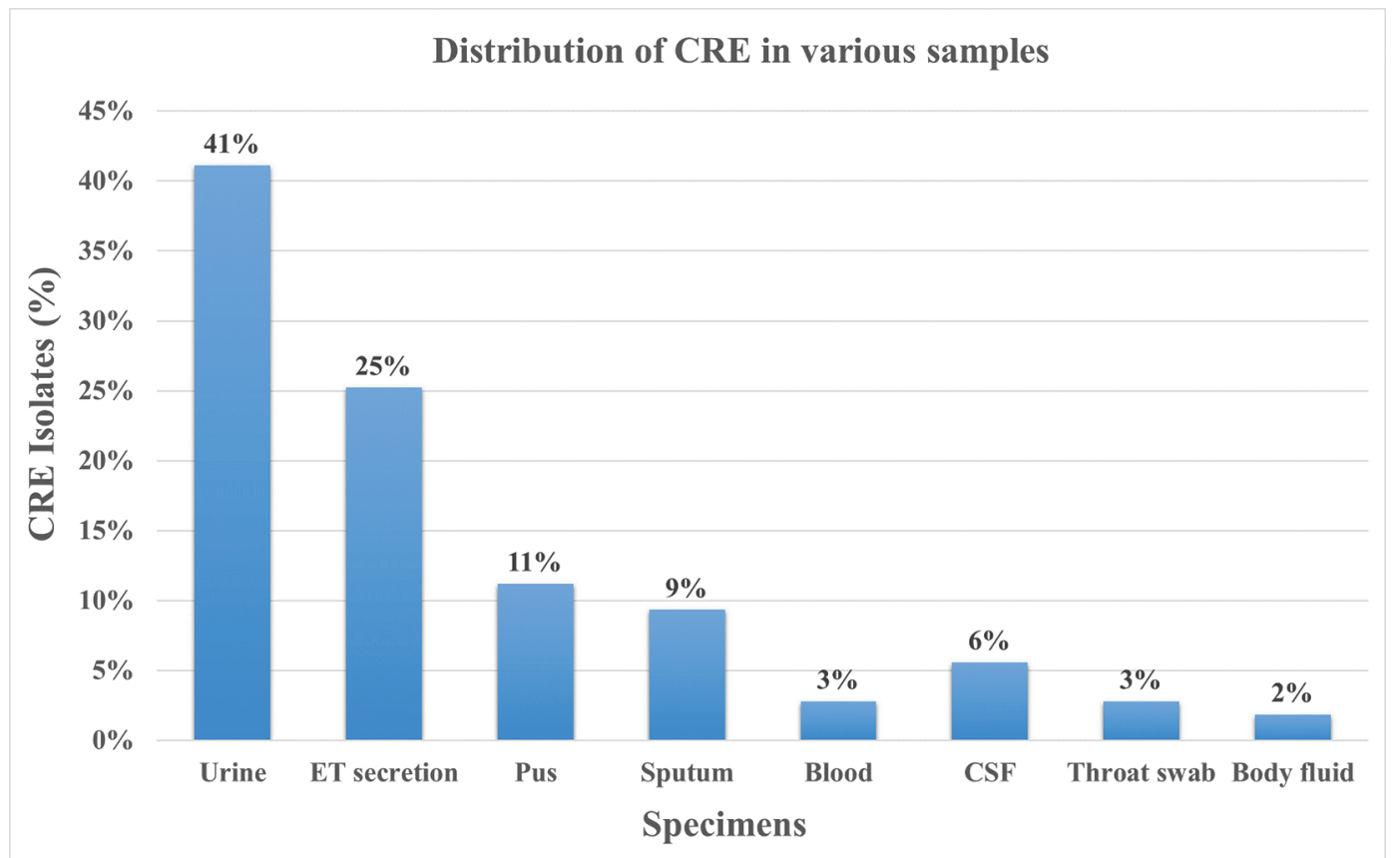

Fig. 3 Distribution of CRE in various clinical samples included in study population. CRE, carbapenem-resistant Enterobacteriaceae.

is 0.081 which concludes no agreement between two test types: MHT and Modified strip CNP test.

\section{Discussion}

Carbapenems are commonly used to treat infections caused by ESBL and/or AmpC $\beta$-lactamases producing and other MDR
Enterobacteriaceae. Although they are stable to hydrolysis by most $\beta$-lactamases, their usage as the last resort antibiotics was seriously compromised by the appearance of new class of bacterial enzymes capable of inactivating carbapenems, called as carbapenemases. ${ }^{28}$

Resistance to carbapenem antimicrobials is either due to expression of carbapenemases or due to combined 
Table 1 Phenotypic detection of CPE by modified Hodge test (MHT) and modified strip CNP test

\begin{tabular}{|l|l|l|l|l|l|}
\hline \multirow{2}{*}{ Isolates } & \multirow{2}{*}{ No. of CRE isolates } & \multicolumn{2}{|c|}{ MHT positive } & \multicolumn{2}{c|}{ CNP positive } \\
\cline { 3 - 6 } & & No. & $\%$ & No. & $\%$ \\
\hline Escherichia coli & 32 & 11 & 34 & 25 & 78 \\
\hline Enterobacter aerogenes & 30 & 12 & 40 & 27 & 90 \\
\hline Enterobacter cloacae & 20 & 06 & 30 & 18 & 90 \\
\hline Klebsiellaspp. & 18 & 13 & 72 & 18 & 100 \\
\hline Citrobacterspp. & 4 & 03 & 75 & 4 & 100 \\
\hline Proteus mirabilis & 2 & 01 & 50 & 1 & 50 \\
\hline Proteus vulgaris & 1 & 00 & 0 & 1 & 100 \\
\hline Total & 107 & 46 & 43 & 94 & 88 \\
\hline
\end{tabular}

Abbreviations: CNP, Carba NP test; CRE, carbapenem-resistant Enterobacteriaceae; MHT, modified Hodge test.

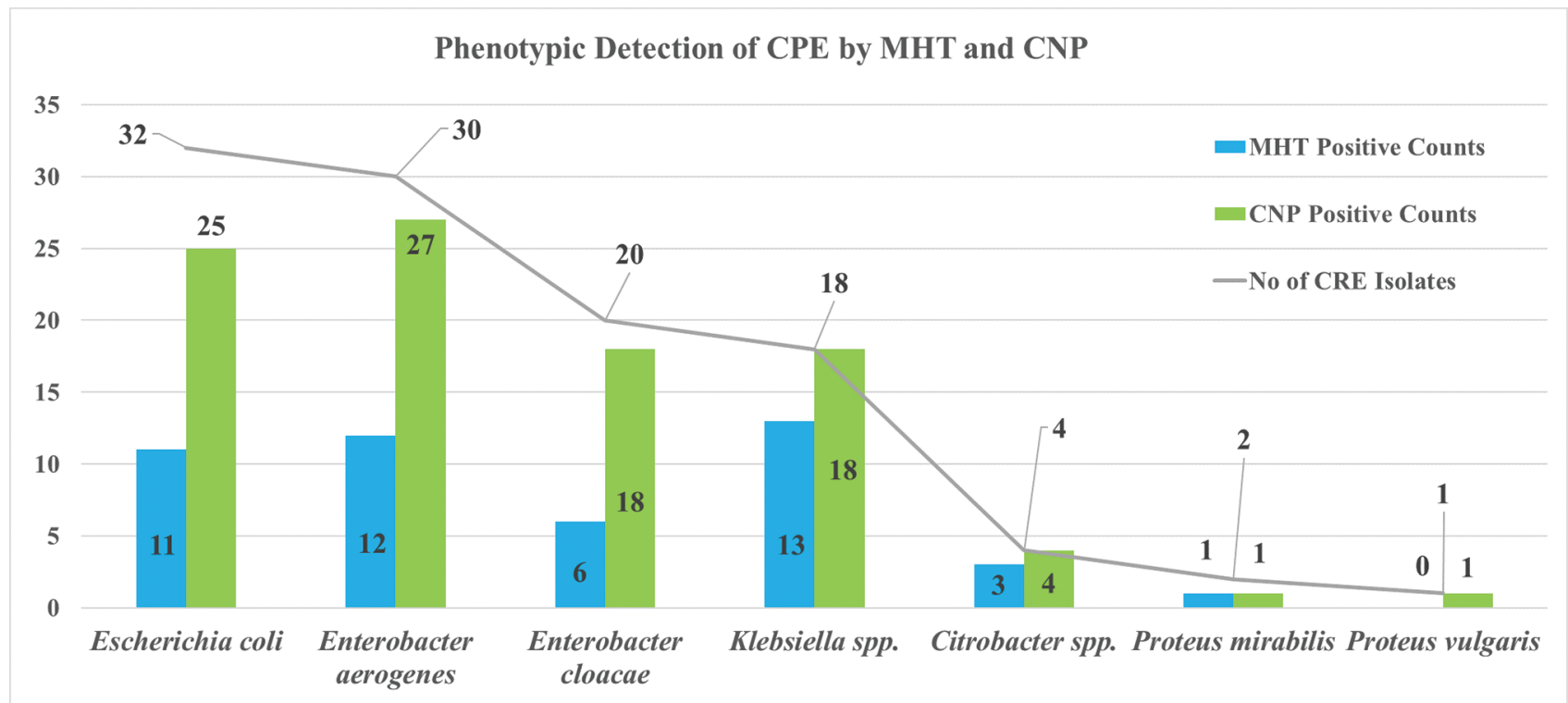

Fig. 4 Phenotypic detection of CPE by MHT and Modified strip CNP test. CNP, Carba NP; CPE, carbapenemase producing Enterobacteriaceae.

mechanisms of resistance (overexpression of broad-spectrum $\beta$-lactamases together with efflux pumps, impermeability); however, carbapenemases production represents the most important mechanism of resistance in Enterobacteriaceae, associated with multi- or pan-drug resistance.

Currently, KPC, MBL, and OXA-48 carbapenemases are considered as major threat in Enterobacteriaceae. They are independently disseminated between different nations worldwide. In India, very few reports on KPC-producing isolates exist. India being the main identified reservoir for NDM type MBL carbapenemases. ${ }^{12-14}$

The last decade is marked with the rapid spread of carbapenemases-producing Enterobacteriaceae worldwide. These infections are often associated with MDR and account for high mortality in patients. Therefore, early detection of CPE is highly crucial not only to guide proper treatment but also to prevent spread of Carbapenem resistance in the society.

In present study, resistance to carbapenem was seen in all age groups and in both the genders. Though in less number, pediatric and geriatric populations were also found to be affected. CRE was isolated from both nosocomial as well community-acquired infection; however, nosocomial spread seems to be the main mode of dissemination (77\% CRE was isolated from IPD and 23\% from OPD). Similar findings were seen in study by Nair and $\mathrm{Vaz}^{29}$ in which most of the CRE isolates were detected in patient samples from the wards (42\%) and the ICU (26\%) followed by OPD patients (19\%). Chauhan et $\mathrm{al}^{30}$ and Pandurangan et $\mathrm{al}^{31}$ also have same observation. Thus, to prevent CRE spread in hospital settings, measures like hand hygiene, contact precautions, minimizing the use of invasive devices, patient and staff cohorting should be given due importance. CRE isolation from samples received in outdoor patient is also of major concern as Enterobacteriaceae mainly colonizes human gut flora; CRE strains can spread rapidly into the community via the fecal oral route. Therefore, screening the contacts of CRE patients and active surveillance testing could possibly help to curtail down the CRE transmission in the community.

CRE was isolated in maximum number from urine (41\%) samples followed by ET secretion (25\%), pus (11\%), sputum (9\%), CSF (6\%), and blood (3\%). The results are consistent with 
Table 2 Comparison of modified strip Carba NP test (CNP) and modified Hodge test (MHT) for detection of CPE

\begin{tabular}{|l|l|l|l|l|l|l|l|}
\hline isolates & CRE & $\begin{array}{l}\text { MHT } \\
\text { positive }\end{array}$ & $\begin{array}{l}\text { CNP } \\
\text { positive }\end{array}$ & $\begin{array}{l}\text { MHT and } \\
\text { CNP positive }\end{array}$ & $\begin{array}{l}\text { MHT } \\
\text { positive- } \\
\text { CNP } \\
\text { negative }\end{array}$ & $\begin{array}{l}\text { MHT negative } \\
\text { and CNP } \\
\text { positive }\end{array}$ & $\begin{array}{l}\text { MHT and CNP } \\
\text { negative }\end{array}$ \\
\hline Escherichia coli & 32 & $11(34 \%)$ & $25(78 \%)$ & $7(22 \%)$ & $4(13 \%)$ & $18(56 \%)$ & $3(9 \%)$ \\
\hline Enterobacter aerogenes & 30 & $12(40 \%)$ & $27(90 \%)$ & $10(33 \%)$ & $2(7 \%)$ & $17(57 \%)$ & $1(3 \%)$ \\
\hline Enterobacter cloacae & 20 & $6(30 \%)$ & $18(90 \%)$ & $5(25 \%)$ & $1(5 \%)$ & $13(65 \%)$ & $1(5 \%)$ \\
\hline Klebsiellaspp. & 18 & $13(72 \%)$ & $18(100 \%)$ & $13(72 \%)$ & $0(0 \%)$ & $5(28 \%)$ & $0(0 \%)$ \\
\hline Citrobacterspp. & 4 & $3(75 \%)$ & $4(100 \%)$ & $3(75 \%)$ & $0(0 \%)$ & $1(25 \%)$ & $0(0 \%)$ \\
\hline Proteus mirabilis & 2 & $1(50 \%)$ & $1(50 \%)$ & $0(0 \%)$ & $1(50 \%)$ & $1(50 \%)$ & $0(0 \%)$ \\
\hline Proteus vulgaris & 1 & $0(0 \%)$ & $1(100 \%)$ & $0(0 \%)$ & $0(0 \%)$ & $1(100 \%)$ & $0(0 \%)$ \\
\hline Total & 107 & $46(43 \%)$ & $94(88 \%)$ & $38(36 \%)$ & $8(7 \%)$ & $56(52 \%)$ & $5(5 \%)$ \\
\hline
\end{tabular}

Abbreviations: CNP, Carba NP test; CRE, carbapenem-resistant Enterobacteriaceae; MHT, modified Hodge test.

studies done by many authors. ${ }^{29-32}$ In all these studies CRE was isolated mainly from urine samples followed by respiratory samples.

In present study, dissemination of carbapenem resistance was seen among all the species of Enterobacteriaceae family as out of 107 CRE isolated, 50 (47\%) were Enterobacter species, 32 (30\%) were E. coli, 18 (17\%) were Klebsiella species, four (4\%) were Citrobacter species, and three (3\%) were Proteus species. Similarly, studies done by other authors also reported interspecies dissemination of carbapenem resistance like Datta et $\mathrm{al}^{33}$ reported carbapenem resistance in $62.12 \%$ of $K$. pneumoniae, $7.58 \%$ of Klebsiella oxytoca, and $16.6 \%$ of E. coli. Bartolini et $\mathrm{al}^{34}$ reported maximum carbapenem resistance in Klebsiella species (88\%) followed by Enterobacter species (8\%) and E. coli (4\%). Pandurangan et $\mathrm{al}^{31}$ observed CRE in E. coli (61\%), K. pneumoniae (21.5\%), and Enterobacter cloacae (6\%). This may be because genes carrying CRE are located on plasmids and transposons causing rapid dissemination of carbapenem resistance. These studies also suggested that CRE were mainly seen in Enterobacter species, E. coli, and Klebsiella species. CDC (2019) has also incorporated these three species in their case definition for surveillance of CP-CRE cases. ${ }^{35}$

CRE isolates were subjected to Modified strip CNP test and MHT for detection of carbapenemase production. Out of 107 CRE isolates, 46 (43\%) were confirmed phenotypically as carbapenemase producer by MHT and 94 (88\%) were confirmed by Modified strip CNP test. There is statistically significant difference in efficiency of MHT and Modified strip CNP test for detection of carbapenemase producing CRE isolates ( $p$-value $<0.05$ ). Modified strip CNP test was more sensitive than MHT for detecting CP-CRE. Our results are consistent with the studies conducted by many authors. The study by Datta et $\mathrm{al}^{33}$ observed CNP test (86.4\% [114/132] positive) shows better result as compared with MHT (75\% [99/132] positive). Similarly, in studies done by Vasoo et $\mathrm{al}^{36}$ and Yamada et $\mathrm{al}^{37} \mathrm{CNP}$ test was found to be more sensitive and specific for detecting CPE as compared with MHT. This may be because Indian subcontinent is endemic for MBL type of carbapenemases mainly NDM..$^{10,12-14}$ and several studies reported MHT has low sensitivity for detecting MBL producers. Bartolini et $\mathrm{al}^{34}$ observed MHT is $100 \%$ sensitive for the detection of KPC but has low sensitivity for VIM and was not able to detect NDM-1. Similarly, Girlich et al ${ }^{21}$ reported that MHT has an excellent sensitivity for detecting KPC and OXA-48 carbapenemases but has low sensitivity (50\%) for NDM-1 producers. EUCAST guidelines ${ }^{38}$ mentioned MHT has low sensitivity for MBL detection. This may be likely due to zinc supplementation in reagents used for Modified strip CNP test which is lacking in MHT. ${ }^{21}$

In present study, measure of agreement (kappa) was 0.081 (no agreement between the two tests). It was in accordance with Datta et $\mathrm{al}^{33}$ who also reported measure of agreement (kappa) value less than 0.001 . Out of 107 CRE isolates, 38 (36\%) isolates showed carbapenemase production by both MHT and CNP tests. Fifty-six isolates (52\%) were CNP test positive but MHT negative, five (5\%) isolates were both MHT and CNP test negative that is they were not identified as carbapenemase producer by either of the test. It may be because these isolates were resistant to carbapenems because of mechanism other than carbapenemase production. Eight (7\%) isolates were MHT positive but CNP test negative; this may be because of two reasons, either MHT gives false positive results as MHT suffers from poor specificity due to false positive results for isolates producing ESBL or AmpC $\beta$-lactamase (AmpC) combined with porin loss as reported by many authors ${ }^{22-24}$ or modified strip CNP test has given false negative results as CNP test has low sensitivity for OXA-48 producers. ${ }^{39}$

CRE isolates are not only resistant to beta lactam antibiotics but also show significant cross resistance between different classes of antibiotics as plasmids carrying genes for carbapenem resistance also carry MDR determinant genes. Infections caused by CRE are resistant to most of the available antibiotics; they are difficult to treat and are therefore associated with major therapeutic failures, increased duration of hospital stays with increased cost of treatment, and high mortality rate. Also, resistance to carbapenems due to carbapenemase production is rapidly transmissible between different species of Enterobacteriaceae, thus early detection of CPE holds high merits to prevent spread of CRE.

Modified strip CNP test shows promising results as compared with MHT. It is simple, inexpensive, reproducible test 
which is easy to perform and interpret and give rapid results in less than 5 minutes. It has high degree of sensitivity and specificity comparable to molecular test. On the other hand, MHT is a cumbersome procedure; results are difficult to interpret and require 24-hour incubation time. Its sensitivity is low especially for MBL producers and also has low specificity.

\section{Conclusion}

CP-CRE have high tendency to spread in the community and are associated with high morbidity and mortality. Modified strip CNP test is a practical solution for early detection of CP-CRE particularly in low resource countries like India which are large reservoir of carbapenemase producers. It can contribute to formulate better treatment plan to avoid therapeutic failures and to design logical infection control policies to prevent further dissemination of carbapenemase producers in the community thus shifting the paradigm of carbapenem resistance.

\section{Authors' Contribution List}

N.P. undertook the research work under the guidance of co-authors, reviewed the literature, did data collection, data analysis, and interpretation, and prepared manuscript. N.V. conceptualized and designed the research work, approved the protocol, guided the research process, provided intellectual input, and proofread the manuscript. S.S. reviewed the literature and did data analysis and interpretation. B.S. provided intellectual input and guidance throughout the research process, did manuscript correction, and revised manuscript for important intellectual content

\section{Financial Disclosure}

None.

\section{Conflict of Interest}

None.

\section{References}

1 Koneman EW. Koneman's color atlas and textbook of Diagnostic Microbiology, Characteristics for Presumptive Identification of Enterobacteriaceae. 7th ed. Philadelphia, PA:Wolters Kluwer;2017:685-689

2 Blair JMA, Webber MA, Baylay AJ, Ogbolu DO, Piddock LJV. Molecular mechanisms of antibiotic resistance. Nat Rev Microbiol 2015;13(1):42-51

3 Peter-Getzlaff S, Polsfuss S, Poledica M, et al. Detection of AmpC beta-lactamase in Escherichia coli: comparison of three phenotypic confirmation assays and genetic analysis. J Clin Microbiol 2011;49(8):2924-2932

4 Papp-Wallace KM, Endimiani A, Taracila MA, Bonomo RA Carbapenems: past, present, and future. Antimicrob Agents Chemother 2011;55(11):4943-4960

5 Yigit $H$, Queenan AM, Anderson GJ, et al. Novel carbapenem-hydrolyzing $\beta$-lactamase, KPC-1, from a carbapenem-resistant strain of Klebsiella pneumoniae. Antimicrob Agents Chemother 2001;45(4):1151-1161

6 World Health Organization. Guidelines for the prevention and control of carbapenem-resistant Enterobacteriaceae,
Acinetobacter baumannii and Pseudomonas aeruginosa in health care facilities. 2017:ISBN 978-92-4-155017-8. Available at: https://www.who.int/infection-prevention/publications/ guidelines-cre/en/. Accessed November 17, 2019

7 Clinical and Laboratory Standards Institute. AST news update. 2017:1(2):9

8 Tille PM. Bailey \& Scotts Diagnostic Microbiology, Expanded Spectrum Cephalosporin Resistance \& Carbapenemase Resistance. 13th ed. St. Louis: Elsevier. ; 2014:326

9 Cornaglia G, Giamarellou H, Rossolini GM Metallo- $\beta$-lactamases: a last frontier for $\beta$-lactams? Lancet Infect Dis 2011;11(5):381-393

10 Nordmann P, Poirel L, Walsh TR, Livermore DM. The emerging NDM carbapenemases. Trends Microbiol 2011;19(12):588-595

11 Poirel L, Potron A, Nordmann P. OXA-48-like carbapenemases: the phantom menace. J Antimicrob Chemother 2012;67(7):1597-1606

12 van Duin D, Doi Y. The global epidemiology of carbapenemase-producing Enterobacteriaceae. Virulence 2017;8(4):460-469

13 Kazi M, Drego L, Nikam C, et al. Molecular characterization of carbapenem-resistant Enterobacteriaceae at a tertiary care laboratory in Mumbai. Eur J Clin Microbiol Infect Dis 2015;34(3):467-472

14 Shanmugam P, Meenakshisundaram J, Jayaraman P. blaKPC gene detection in clinical isolates of carbapenem resistant Enterobacteriaceae in a tertiary care hospital. J Clin Diagn Res 2013;7(12):2736-2738

15 Nordmann P, Poirel L, Dortet L. Rapid detection of carbapenemase-producing Enterobacteriaceae. Emerg Infect Dis 2012;18(9):1503-1507

16 Poirel L, Walsh TR, Cuvillier V, Nordmann P. Multiplex PCR for detection of acquired carbapenemase genes. Diagn Microbiol Infect Dis 2011;70(1):119-123

17 Cuzon G, Naas T, Bogaerts P, Glupczynski Y, Nordmann P. Evaluation of a DNA microarray for the rapid detection of extended-spectrum $\beta$-lactamases (TEM, SHV and CTX-M), plasmid-mediated cephalosporinases (CMY-2-like, DHA, FOX, ACC-1, ACT/MIR and CMY-1-like/MOX) and carbapenemases (KPC, OXA-48, VIM, IMP and NDM). J Antimicrob Chemother 2012;67(8):1865-1869

18 Hrabák J, Walková R, Studentová V, Chudácková E, Bergerová T. Carbapenemase activity detection by matrix-assisted laser desorption ionization-time of flight mass spectrometry. J Clin Microbiol 2011;49(9):3222-3227

19 Lee W, Chung HS, Lee Y, et al. Comparison of matrix-assisted laser desorption ionization-time-of-flight mass spectrometry assay with conventional methods for detection of IMP-6, VIM-2, NDM1,SIM-1, KPC-1, OXA-23, and OXA-51 carbapenemase-producing Acinetobacter spp., Pseudomonas aeruginosa, and Klebsiella pneumoniae. Diagn Microbiol Infect Dis 2013;77(3):227-230

20 Poirel L, Nordmann P. Rapidec Carba NP Test for rapid detection of carbapenemase producers. J Clin Microbiol 2015;53(9):3003-3008

21 Girlich D, Poirel L, Nordmann P. Value of the modified Hodge test for detection of emerging carbapenemases in Enterobacteriaceae. J Clin Microbiol 2012;50(2):477-479

22 Carvalhaes CG, Picão RC, Nicoletti AG, Xavier DE, Gales AC. Cloverleaf test (modified Hodge test) for detecting carbapenemase production inKlebsiella pneumoniae: be aware of false positive results. J Antimicrob Chemother 2010;65(2):249-251

23 Anderson KF, Lonsway DR, Rasheed JK, et al. Evaluation of methods to identify the Klebsiella pneumoniae carbapenemase in Enterobacteriaceae. J Clin Microbiol 2007;45(8):2723-2725

24 Mochon AB, Garner OB, Hindler JA, et al. New Delhi metallo- $\beta$-lactamase (NDM-1)-producing Klebsiella pneumoniae: case report and laboratory detection strategies. J Clin Microbiol 2011;49(4):1667-1670 
25 Srisrattakarn A, Lulitanond A, Wilailuckana C, et al. Modification and evaluation of the Carba NP test by use of paper strip for simple and rapid detection of carbapenemase-producing Enterobacteriaceae. World J Microbiol Biotechnol 2016;32(7):117

26 Ho PL, Wang Y, Wing-Sze Tse C, et al. Rapid detection of carbapenemase production in Enterobacteriaceae by use of a modified paper strip Carba NP method. J Clin Microbiol 2017;56(1):e01110-e01117

27 Centersfor Disease Control and Prevention. Modified Hodge test for carbapenemase detection in Enterobacteriaceae. Available at: https://www.cdc.gov/HAI/pdfs/labSettings/ HodgeTest_Carbapenemase_Enterobacteriaceae.pdf. Accessed November 17, 2019

28 Bedenić B, Sardelić S. Textbook on Carbapenemases. London:Intech Open;2018:1-12

29 Nair PK, Vaz MS. Prevalence of carbapenem resistant Enterobacteriaceae from a tertiary care hospital in Mumbai, India. J Microbiol Inf Dis 2013;3(4):207-210

30 Chauhan K, Pandey A, Asthana AK, Madan M. Evaluation of phenotypic tests for detection of Klebsiella pneumoniae carbapenemase and metallo-beta-lactamase in clinical isolates of Escherichia coli and Klebsiella species. Indian J Pathol Microbiol 2015;58(1):31-35

31 Pandurangan S, BegumEsak S, Narayanasamy A, et al. Phenotypic detection methods of carbapenemase production in Enterobacteriaceae. Int J Curr Microbiol Appl Sci 2015;4(6):547-552

32 Mangayarkarasi V, Moses SP, Swarna SR, et al. In-house standardization of Carba NP test for carbapenemase detection in gram negative bacteria. Int J Curr Microbiol Appl Sci 2018;7(1):2876-2881
33 Datta S, Dey R, Dey JB, Ghosh S. A comparative study of modified Hodge test and Carba NP test for detecting carbapenemase production in gram-negative bacteria. Med J Dr D.Y. Patil Uni. 2017;10:365-369

34 Bartolini A, Frasson I, Cavallaro A, Richter SN, Palù G. Comparison of phenotypic methods for the detection of carbapenem non-susceptible Enterobacteriaceae. Gut Pathog 2014;6:13

35 Centers for Disease Control and Prevention. Case definition for surveillance of CP-CRE; 2018. Available at: https://wwwn. cdc.gov/nndss/conditions/carbapenemase-producing-carbapenem-resistant-enterobacteriaceae/case-definition/2018/. Accessed November 17, 2019

36 Vasoo S, Cunningham SA, Kohner PC, et al. Comparison of a novel, rapid chromogenic biochemical assay, the Carba NP test, with the modified Hodge test for detection of carbapenemase-producing Gram-negative bacilli. J Clin Microbiol 2013;51(9):3097-3101

37 Yamada K, Kashiwa M, Arai K, Nagano N, Saito R. Comparison of the Modified-Hodge test, Carba NP test, and carbapenem inactivation method as screening methods for carbapenemase-producingEnterobacteriaceae. J Microbiol Methods 2016;128:48-51

38 Giske CG, Martinez-Martinez L, Cantón R, Stefani S, Skov R, Glupczynski Y, et al. European Committee on Antimicrobial Susceptibility Testing. Guidelines for detection of resistance mechanisms and specific resistances of clinical and/or epidemiological importance. 2017;2:1-43

39 Tijet N, Boyd D, Patel SN, Mulvey MR, Melano RG. Evaluation of the Carba NP test for rapid detection of carbapenemase-producing Enterobacteriaceae and Pseudomonas aeruginosa. Antimicrob Agents Chemother 2013;57(9):4578-4580 